\title{
FAULT DETECTION BASED ON LYAPUNOV EXPONENTS ESTIMATION FOR STABILIZED MECHANICAL SYSTEMS
}

\author{
VOLODYMYR PUZYROV \\ Vasyl Stus Donetsk National University, Faculty of Mathematics and Information Technology, Vinnytsya, Ukraine \\ Leonardo Acho, Gisela Pujol, José Rodellar \\ Universitat Politècnica de Catalunya-BarcelonaTech (EEBE), Department of Mathematics, Barcelona, Spain \\ e-mail: gisela.pujol@upc.edu
}

\begin{abstract}
We study a stabilizable mechanical system in the vicinity of an equilibrium position. This position, as a rule, is unstable, and the system is underactuated. It is assumed that faults affect the technical process and its control. We suggest a fault diagnosis technique based on estimation of Lyapunov characteristic exponents of measured variables. A model of a linear switching system is involved for the system with faults description, and a common quadratic Lyapunov function is used to evaluate the deviation of the maximum exponent with respect to the default system. A scheme of fault magnitude estimation is suggested related to the degree of this deviation. An example of a 2-degree of freedom system is presented to illustrate the procedure.
\end{abstract}

Keywords: fault detection, switched system, Lyapunov characteristic exponent, underactuated system

\section{Introduction}

Fault detection systems (FDS) are successfully used in many types of technical processes to improve reliability and safety (Frank, 1990; Isermann, 1997, 2005, 2011; Gertler, 1998; Chen and Patton, 1999; Ding, 2008). Recently, improved methods for detecting malfunctions have been applied to numerous technical and engineering objects, such as machine tools, industrial robots, automobile power trains and many others. The using of FDS entails the benefits of good operational safety due to its key features of an early warning system and cost effectiveness, since condition-based maintenance is performed despite corrective or preventive maintenances. The main tasks of FDS are: fault detection (FD) to recognize that a fault happened, fault isolation or diagnosis (FDI) to find the location and cause of the fault, and fault estimation. Advanced FD methods are based on a mathematical process and signal models.

Model-based FD methods use residuals which indicate changes between the process and the model. One general assumption is that the residuals have changed significantly, so that detection is possible with regards to the mostly inherent stochastic element. This means that the deviation of the residual after the appearance of a fault is large enough and lasts long enough to be detected. It is usually assumed that some general features are presented here:

- parity equations - model structure and parameters are known and fit the process well;

- state estimation (state observers) - methods based on state observers are still a topic of active research. In this approach, the residual is the observation error calculated from the process measurements and the output of the reference model;

- parameter estimation (small changes are detectable for slowly and fast progressing faults) - parameter estimation methods are well established and widely used in control systems, e.g., in adaptive controllers. They have also been successfully applied to a variety of fault detection problems where sufficiently accurate linear process models could be derived. 
In the signal processing based FDI, some mathematical or statistical operations are performed on the measurements to extract the information about the fault (Saberi et al., 2007; Zhang, 2010). Also FD methods based on time- and frequency-domain signal analysis without an explicit mathematical model are state of the art in process supervision. In this approach, fault indicators are derived from process measurements via limit and trend checking of the process signals and by means of various spectral analysis methods (wavelet analysis, sidebands etc.).

As well, other approaches and techniques have been employed for the last decades. Among them the geometrical approach, expert system approach, fuzzy techniques and neural networks are presented (Talebi et al., 2010; Meskin and Khorasani, 2011).

The main objective of this work is to detect when a measurement failure has occurred, proposing a time interval of the occurrence. To do that, we consider a known failure bound but failures are not constant all along the experimental time. To deal with this problem, we suppose that failures can be collected and considered as an added disturbance, which takes values on a set of unknown (but bounded) parameters. Then, once the system is stabilized (the control law can be freely determined by the user), we use our main result to define the time interval of failure occurrence. In this scheme, we use switching theory due to possible values of failures, and Lyapunov exponents theory to determine stability. We consider that failure measurement implies instability. So, fault detection is based on the loss of stability of the controlled system.

Moreover, the control design must be able to maintain stability despite sensors faults, considered bounded. The procedure is based on linearized equations around a non-stable equilibrium point, with measurement faults. The sensor faults induce errors on the measurements, so derivation of the state variable $x(t)$ is $\Delta x(t)$. To detect it, we split the time interval and fix the allowed maximum fault. Then, we estimate when this bound is exceeded. The multiplicative model is used: $\Delta x(t)=E(\delta) x(t)$, where $E(\delta)$ is the faulty matrix. We want to estimate the time interval when a fault occurs. Then, in each time interval $\left[t_{j}, t_{j+1}\right]$, the expression for $E(\delta)$ becomes $E\left(\delta_{j}\right):=E_{j}$ (see Fig. 1).

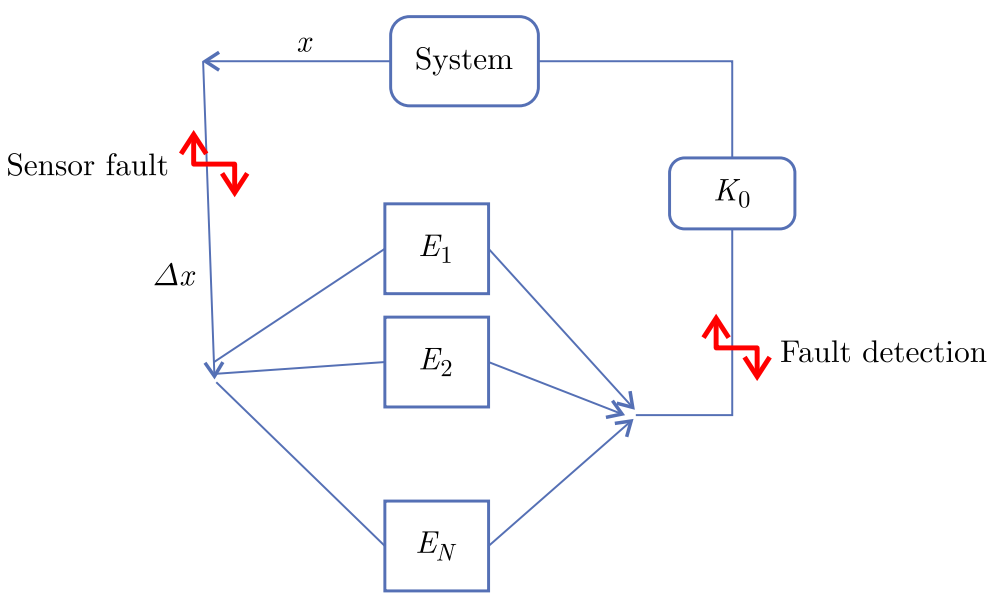

Fig. 1. Schematic diagram of the faulty switched system

Consider mechanical systems described by deterministic analytical models whose dynamics are determined by Lagrange equations. We assume that a control function is presented, and the number of degrees of freedom exceeds the dimension of the vector of control generalized forces (underactuated systems). The research of such systems is of great importance for various applications, since in practice it is often necessary to reduce the number of motor elements that carry out control. For example, in astronautics the equipment of each link of the robot manipulator with an electric motor entails an increase in the weight and cost of the spacecraft as well as a decrease in the payload weight. Moreover, if control algorithms of such a robot are based on the mandatory use of all engines, the failure of one of them means the failure of the 
entire robot. This can be avoided by developing manipulator control laws that are effective for one or more idle engines, that is, solving corresponding control tasks with a shortage of control actions.

In addition to FD method, the paper involves two topics:

a) Switched systems (Hassibi and Boyd, 1998; Shorten and Narendra, 1998; Liberzon and Morse, 1999; Zhai et al., 2000; Liberzon, 2003; Xie and Wang, 2003; Geromel and Colaneri, 2006). Switched systems are of variable structure or multi-modal class. According to (Liberzon and Morse, 1999), a switched system can be viewed as an hybrid dynamical system that is composed of a family of continuous-time subsystems along with a switching law among them.

b) Characteristic Lyapunov exponents (CLE). An important characteristic in the analysis of dynamic systems is finding of the highest Lyapunov exponent, as well as the full spectrum. The Lyapunov exponents are a quantitative measure of the sensitivity of phase trajectories to initial conditions. There are several methods for calculating Lyapunov exponents (Shimada and Nagashima, 1979; Molchanov and Pyatnitskii, 1989; Muller, 1995). When the equations of motion of the dynamical system are known, there exist well designed techniques for computing them. Also, there are numerous results on calculation of CLE from experimental data (Wolf et al., 1985; Eckmann and Ruelle, 1992).

The aim of the paper is the design of an algorithm which allows one to detect faults in systems under study based on estimation of the maximal CLE value for the output signal of the state vector.

\section{Preliminaries}

\subsection{System equations}

In this paper, we deal with autonomous (time-invariant) mechanical systems defined by equations of motion in the Lagrange form. Consider the general coordinates $\mathbf{q}=\left[q_{1}, \ldots, q_{n_{1}}\right]^{\mathrm{T}}$, and the control input $\mathbf{u}(t)=\left[u_{1}(t), \ldots, u_{m_{1}}(t), \ldots, u_{n_{1}}(t)\right]^{\mathrm{T}}$ with $u_{s} \equiv 0$ for $1 \leqslant s \leqslant m_{1}<n_{1}$. Then, the nonlinear system is obtained from

$$
\frac{d}{d t} \frac{\partial \mathcal{K}}{\partial \dot{q}_{s}}-\frac{\partial \mathcal{K}}{\partial q_{s}}=\frac{\partial \Pi}{\partial q_{s}}+Q_{s}+u_{s}(t) \quad s=\overline{1, n_{1}}
$$

where $\mathcal{K}, \Pi$ are the kinetic and potential energies of the system, $Q_{s}$ represent the generalized non-potential forces, and $u_{s}(t)$ are controls. We assume that all coordinates $q_{s}$ are positional (noncyclic) $^{1}$ and system $(2.1)$ with $\mathbf{u}(t) \equiv \mathbf{0}$ has an unstable equilibrium

$$
\mathbf{q}=\mathbf{q}_{0} \quad \dot{\mathbf{q}}=\mathbf{0}
$$

which is stabilizable with an appropriate control function.

Let us consider a state space model of the uncontrolled nonlinear dynamical system

$$
\dot{\mathbf{x}}=\mathbf{F}(t, \mathbf{x}) \quad \mathbf{F}(t, \mathbf{0})=\mathbf{0}
$$

where the function $\mathbf{F}$ is bounded on time and continuously differentiable. Also a closed-loop controlled system

$$
\begin{array}{lll}
\dot{\mathbf{x}}=\mathbf{F}(t, \mathbf{x})+\mathbf{B}(t, \mathbf{x}) \mathbf{u}(t) & \mathbf{B}(t, \mathbf{0})=\mathbf{0} & \\
\mathbf{y}=\mathbf{C}(t, \mathbf{x})+\mathbf{D}(t, \mathbf{x}) \mathbf{u}(t) & \mathbf{C}(t, \mathbf{0})=\mathbf{0} & \mathbf{D}(t, \mathbf{0})=\mathbf{0}
\end{array}
$$

\footnotetext{
${ }^{1}$ While cyclic coordinates are in, we use the Routh function instead of Lagrangian.
} 
is associated with (2.3), and input-output behaviour is considered for (2.4). Here $\mathbf{u}$ is the vector of control inputs, and the output $\mathbf{y}$ represents measurements. If the control function is designed to stabilize the equilibrium $\mathbf{x}=\mathbf{0}$, then in some neighbourhood of the origin the system behaviour may be approximated by the linearized equations

$$
\dot{\mathbf{x}}=\mathbf{F}_{0}(t) \mathbf{x}+\mathbf{B}_{0}(t) \mathbf{u}(t) \quad \mathbf{y}=\mathbf{C}_{0}(t) \mathbf{x}+\mathbf{D}_{0} \mathbf{u}(t)
$$

where

$$
\mathbf{F}_{0}=\left.\frac{\partial \mathbf{F}(t, \mathbf{x})}{\partial \mathbf{x}}\right|_{\mathbf{x}=\mathbf{0}} \quad \mathbf{B}_{0}=\mathbf{B}(t, \mathbf{0}) \quad \mathbf{C}_{0}=\left.\frac{\partial \mathbf{C}(t, \mathbf{x})}{\partial \mathbf{x}}\right|_{\mathbf{x}=\mathbf{0}} \quad \mathbf{D}_{0}=\mathbf{B}(t, \mathbf{0})
$$

We recall that the following statements are valid (Muller, 2009):

Theorem 2.1. If the linearized system $\dot{\mathbf{x}}=\mathbf{F}_{0} \mathbf{x}$ is exponentially stable, and

$$
\lim _{\mathbf{x} \rightarrow \mathbf{0}} \frac{\left\|\mathbf{F}(t, \mathbf{x})-\mathbf{F}_{0} \mathbf{x}\right\|}{\|\mathbf{x}\|}=0
$$

holds, then the equilibrium point of nonlinear system (2.3) is exponentially stable as well.

Theorem 2.2. If the linearized system $\left.(2.5)_{1}\right)$ is stabilized exponentially by a linear static or dynamic output feedback $\left.(2.5)_{2}\right)$, and

$$
\lim _{\mathbf{x} \rightarrow \mathbf{0}} \frac{\left\|\mathbf{C}(t, \mathbf{x})-\mathbf{C}_{0} \mathbf{x}\right\|}{\|\mathbf{x}\|}=0
$$

holds, then the equilibrium point of nonlinear system (2.4) is exponentially stabilized by the same feedback.

We suppose that equations (2.1) satisfy conditions of Theorems 2.1 and 2.2. Then using $\widetilde{\mathbf{q}}=\mathbf{q}-\mathbf{q}_{0}$, we obtain

$$
\mathbf{M} \ddot{\widetilde{\mathbf{q}}}+\mathbf{G}_{0} \dot{\overrightarrow{\mathbf{q}}}+\mathbf{H}_{0} \widetilde{\mathbf{q}}=\mathbf{u}
$$

Here $\mathbf{M}, \mathbf{G}_{0}, \mathbf{H}_{0}$ are constant matrices, $\mathbf{M}$ is symmetric and positive definite. Since $\mathbf{M}$ is nonsingular, taking $\mathbf{x}(t)=[\dot{\tilde{\mathbf{q}}}(t), \widetilde{\mathbf{q}}(t)]^{\mathrm{T}}$, system (2.8) may be rewritten in the form

$$
\dot{\mathbf{x}}(t)=\mathbf{A}_{0} \mathbf{x}(t)+\mathbf{B u}(t)
$$

where the matrix $\mathbf{A}_{0}$ is an unstable square matrix of the order $n=2 n_{1}$. We note that in some situations the use of equations (2.8) is more convenient. For instance, when system contains uncertain parameters or is of a high order, operating with matrices of half size is welcomed.

\subsection{Switching systems}

Besides that, we use the model of autonomous switched systems described by

$$
\dot{\mathbf{x}}(t)=\mathbf{A}_{\sigma(t)} \mathbf{x}(t)+\mathbf{B}_{\sigma(t)} \mathbf{u}(t) \quad \mathbf{x}\left(t_{0}\right)=\mathbf{x}_{0}
$$

defined for all $t \geqslant 0$ where $\mathbf{x}(t) \in R^{n}$ is the state vector, $\mathbf{u}(t) \in R^{q}$ is the control, $\sigma(\cdot): R \rightarrow$ $\{1,2, \ldots, N\}$ is a piecewise constant function (usually called the switching rule), and where the matrices $\mathbf{A}_{\sigma(t)}, \mathbf{B}_{\sigma(t)}$ are constructed by switching between a set of the constant matrices $\mathbf{A}_{\sigma(t)} \in\left\{\mathbf{A}_{1}, \mathbf{A}_{2}, \ldots, \mathbf{A}_{m}\right\}, \mathbf{B}_{\sigma(t)} \in\left\{\mathbf{B}_{1}, \mathbf{B}_{2}, \ldots, \mathbf{B}_{m}\right\}, \mathbf{A}_{j} \in R^{n \times n}, \mathbf{B}_{j} \in R^{n \times q}(j=\overline{1, N})$, i.e. at 
any time $t$ the matrices $\mathbf{A}_{\sigma(t)}, \mathbf{B}_{\sigma(t)}$ are equal to one and only one pair of the matrices $\mathbf{A}_{j}, \mathbf{B}_{j}$ in the above sets.

It is supposed that the solution $\mathbf{x}\left(t, \mathbf{x}_{0}\right)$ is continuous, so if the pair $\mathbf{A}_{i}, \mathbf{B}_{i}$ is associated with system (2.10) in the time interval $\left[t_{1}, t_{2}\right)$, and the pair $\mathbf{A}_{j}, \mathbf{B}_{j}$ in the time interval $\left[t_{2}, t_{3}\right)$, there is no gap on the phase trajectory while switching. Thus, the initial state $x\left(t_{2}\right)$ in the interval $\left[t_{2}, t_{3}\right)$ is taken the terminal state (just before switching) of the vector $\mathbf{x}(t)$ in the previous time interval. Also, we involve that once the matrices $\mathbf{A}_{\sigma(t)}, \mathbf{B}_{\sigma(t)}$ assume the values $\mathbf{A}_{j}, \mathbf{B}_{j}$ for an interval of time $\tau$ where $\tau \geqslant \tau_{\star}>0$, and the constant $\tau_{\star}$ is arbitrarily small and independent of $j$.

It is well known that if all the matrices $\mathbf{A}_{j}$ are stable (have spectrum in the open left half of the complex plane) and share a common quadratic Lyapunov function (CQLF), then switched system (2.10) is globally exponentially stable (uniformly with respect to switching signals). In other words, if there exist two symmetric positive definite matrices $\mathbf{P}$ and $-\mathbf{Q}$ such that

$$
\mathbf{A}_{j}^{\mathrm{T}} \mathbf{P}+\mathbf{P A}_{j} \leqslant \mathbf{Q} \quad j=\overline{1, N}
$$

then there exist constants $\alpha \geqslant 1$ and $\lambda>0$ that solution of (2.10) satisfies

$$
\|\mathbf{x}(t)\| \leqslant \alpha \mathrm{e}^{-\lambda t}\|x(0)\|
$$

\section{Main results}

When the mechanical system is working around the equilibrium state, its behaviour can be described by a linear time invariant (LTI) model of the form

$$
\dot{\mathbf{x}}(t)=\mathbf{A}_{0} \mathbf{x}(t)+\mathbf{B}_{0} \mathbf{u}(t)+\mathbf{F}_{1} \mathbf{f}(t)
$$

Here $\mathbf{x}(t)$ is the state vector, $\mathbf{u}(t)$ is the vector of manipulated inputs (known), and $\mathbf{f}(t)$ is the vector of faults.

\subsection{Controlled system without faults}

For "ideal" faultless system $\mathbf{f}(t) \equiv \mathbf{0}$.

Suppose that $\left(\mathbf{A}_{0}, \mathbf{B}_{0}\right)$ is stabilizable, $\left(\mathbf{C}_{0}, \mathbf{A}_{0}\right)$ detectable, and we synthesize the control $\mathbf{u}(t)=\mathbf{K}_{0} \mathbf{x}(t)$ that stabilizes the faultless system. We assume a state-feedback controller $\mathbf{K}_{0}$ has a complete access to the state vector, and velocities are calculated according to $\mathbf{q}(t)$ change. Thus, the nominal system takes the form

$$
\dot{\mathbf{x}}(t)=\mathbf{A x}(t) \quad \mathbf{A}=\mathbf{A}_{0}+\mathbf{B}_{0} \mathbf{K}_{0}
$$

\section{2. $\quad$ Fault detection as switching}

We suppose now that the system presents measurement faults.

To detect faults we shall use the classical state observer scheme, i.e. the faults will be modeled as state variable changes $\Delta \mathbf{x}(t)$. In this paper, we have limited ourselves to sensor and actuator faults. To study the behaviour of $\mathbf{x}(t)$ in some finite time interval $\left[t_{1}, t_{1}+T\right]$, we split this interval into small parts $\left[t_{j}, t_{j+1}\right]\left(t_{j+1}-t_{j}=h_{j} \geqslant h>0, j=\overline{1, N}, t_{N+1}=t_{1}+T\right)$, and assume that $\mathbf{E}(\delta)$ is a piecewise function, and in each interval the replacement $\Delta \mathbf{x}(t)$ is allowed. With this, we come to the switching system

$$
\dot{\mathbf{x}}(t)=\left(\mathbf{A}+\mathbf{E}\left(\boldsymbol{\delta}_{j}\right)\right) \mathbf{x}(t) \quad \mathbf{E}(\mathbf{0})=\mathbf{0}
$$

where $\mathbf{E}_{j}=\mathbf{E}\left(\boldsymbol{\delta}_{j}\right)$ is the faulty matrix in the time interval $\left[t_{j}, t_{j+1}\right]$. 


\subsection{Stability theorem}

We present first an instrumental lemma.

Lemma 3.1. Consider a square nonsingular complex matrix $\mathbf{W}=\left\{w_{s k}\right\}$ of the order $n$ and the matrix $\boldsymbol{\Psi}=\operatorname{diag}\left(\psi_{1}, \ldots, \psi_{n}\right), 0<\psi_{1} \leqslant \psi_{2} \leqslant \cdots \leqslant \psi_{n}$. Then real the matrices $\mathbf{W} \boldsymbol{\Psi} \mathbf{W}^{\star}-\psi_{1} \mathbf{W} \mathbf{W}^{\star}$ and $\psi_{n} \mathbf{W} \mathbf{W}^{\star}-\mathbf{W} \boldsymbol{\Psi} \mathbf{W}^{\star}$ are positive semidefinite.

Proof. Let us introduce the auxiliary matrix $\Delta \boldsymbol{\Psi}=\operatorname{diag}\left(0, \psi_{2}-\psi_{1}, \ldots, \psi_{n}-\psi_{1}\right)=$ $\operatorname{diag}\left(0, \alpha_{1}^{2}, \ldots, \alpha_{n-1}^{2}\right) . \alpha_{s} \geqslant 0, s=\overline{1, n-1}$, and $\mathbf{I}$ is identity matrix. Then the following implications take place

$$
\begin{gathered}
\mathbf{W} \boldsymbol{\Psi} \mathbf{W}^{\star}-\psi_{1} \mathbf{W} \mathbf{W}^{\star}=\mathbf{W}\left(\boldsymbol{\Psi}-\psi_{1} \mathbf{I}_{n}\right) \mathbf{W}^{\star}=\mathbf{W} \Delta \boldsymbol{\Psi} \mathbf{W}^{\star} \\
=\mathbf{W} \Delta \boldsymbol{\Psi}^{1 / 2} \Delta \boldsymbol{\Psi}^{1 / 2} \mathbf{W}^{\star}=\left(\mathbf{W} \Delta \boldsymbol{\Psi}^{1 / 2}\right)\left(\mathbf{W} \Delta \boldsymbol{\Psi}^{1 / 2}\right)^{\star}
\end{gathered}
$$

The last matrix product may be interpreted as Gramian matrix for system of vectors

$$
\left[\alpha_{1} w_{12}+\cdots+\alpha_{n-1} w_{1 n}\right], \ldots,\left[\alpha_{1} w_{n 2}+\cdots+\alpha_{n-1} w_{n n}\right]
$$

hence it is non-negative. The part of the statement concerning the matrix $\psi_{n} \mathbf{W} \mathbf{W}^{\star}-$ $\mathbf{W} \boldsymbol{\Psi} \mathbf{W}^{\star}$ may be proven in the same manner.

Now we state the following theorem.

Theorem 3.1. Suppose that the given matrix A in system (3.3) is stable, has no multiple eigenvalues, and the matrix $\mathbf{S}$ brings it to a Jordan normal form $\mathbf{J}=\mathbf{S}^{-1} \mathbf{A S}$. Let further its maximum CLE be equal to $\lambda^{\max }$, and $\left\|\boldsymbol{\delta}_{j}\right\| \leqslant \delta_{0}>0$ for all $j \in\{1, \ldots, N\}$. Then:

(i) If $\delta_{0}$ is small enough with $\delta_{0}<\left|\lambda^{\max }\right|$, system (3.3) is globally exponentially stable (uniformly on $j$ ) with the common quadratic Lyapunov function (CQLF)

$$
V(\mathbf{x})=\mathbf{x}^{\mathrm{T}}\left(\mathbf{S}^{-1}\right)^{\mathrm{T}} \mathbf{S}^{-1} \mathbf{x}
$$

(ii) Let $\lambda_{j}$ be the maximum eigenvalue of the matrix $\left(\mathbf{S}^{-1}\right)^{\mathrm{T}} \mathbf{S}^{-1} \mathbf{E}_{j}+\mathbf{E}_{j}^{\mathrm{T}}\left(\mathbf{S}^{-1}\right)^{\mathrm{T}} \mathbf{S}^{-1}$. If

$$
\left.\sup \lambda_{j}\right|_{l_{1} \leqslant j \leqslant l_{2}}=\tilde{\lambda} \quad 1 \leqslant l_{1}, \quad l_{2} \leqslant N
$$

then in the time interval $\left(t_{l_{1}}, t_{l_{2}}\right)$ the inequality

$$
\|\mathbf{x}(t)\| \leqslant \alpha \exp \left[\left(\lambda^{\max }+\frac{1}{2} \tilde{\lambda}\right) t\right]\left\|\mathbf{x}\left(t_{l_{1}}\right)\right\|
$$

holds.

Note. A little bit more realistic is to affirm that its maximum CLE does not exceed $\lambda$, i.e. is equal to $\lambda-\varepsilon$, where $\varepsilon>0$ characterizes the calculation error.

Proof. Consider a quadratic Lyapunov function with $\mathbf{P}=\left(\mathbf{S}^{-1}\right)^{\mathrm{T}} \mathbf{S}^{-1}$. Notice that

$$
\begin{aligned}
& \mathbf{A}^{\mathrm{T}}\left(\mathbf{S}^{-1}\right)^{\mathrm{T}} \mathbf{S}^{-1}+\left(\mathbf{S}^{-1}\right)^{\mathrm{T}} \mathbf{S}^{-1} \mathbf{A}=\left(\left(\mathbf{S}^{\mathrm{T}}\right)^{-1} \mathbf{S}^{\mathrm{T}}\right) \mathbf{A}^{\mathrm{T}}\left(\mathbf{S}^{-1}\right)^{\mathrm{T}} \mathbf{S}^{-1}+\left(\mathbf{S}^{-1}\right)^{\mathrm{T}} \mathbf{S}^{-1} \mathbf{A}\left(\mathbf{S} \mathbf{S}^{-1}\right) \\
& \quad=\left(\mathbf{S}^{\mathrm{T}}\right)^{-1}\left(\mathbf{S}^{\mathrm{T}} \mathbf{A}^{\mathrm{T}}\left(\mathbf{S}^{-1}\right)^{\mathrm{T}}\right) \mathbf{S}^{-1}+\left(\mathbf{S}^{-1}\right)^{\mathrm{T}}\left(\mathbf{S}^{-1} \mathbf{A} \mathbf{S}\right) \mathbf{S}^{-1}=\left(\mathbf{S}^{\mathrm{T}}\right)^{-1} \mathbf{J}^{\mathrm{T}} \mathbf{S}^{-1}+\left(\mathbf{S}^{-1}\right)^{\mathrm{T}} \mathbf{J} \mathbf{S}^{-1} \\
& \quad=\left(\mathbf{S}^{-1}\right)^{\mathrm{T}}\left(\mathbf{J}+\mathbf{J}^{\mathrm{T}}\right) \mathbf{S}^{-1}
\end{aligned}
$$

and this matrix does not exceed $2 \lambda^{\max }\left(\mathbf{S}^{-1}\right)^{\mathrm{T}} \mathbf{S}^{-1}$. By (3.3), the time derivative of $V$ is a quadratic form with matrix

$$
\begin{aligned}
& \left(\mathbf{S}^{-1}\right)^{\mathrm{T}}\left(\mathbf{J}+\mathbf{J}^{\mathrm{T}}\right) \mathbf{S}^{-1}+\mathbf{E}_{j}^{\mathrm{T}}\left(\mathbf{S}^{-1}\right)^{\mathrm{T}} \mathbf{S}^{-1}+\left(\mathbf{S}^{-1}\right)^{\mathrm{T}} \mathbf{S}^{-1} \mathbf{E}_{j} \\
& \quad \leqslant\left(\lambda^{\max } \mathbf{I}+\mathbf{E}_{j}^{\mathrm{T}}\right)\left(\mathbf{S}^{-1}\right)^{\mathrm{T}} \mathbf{S}^{-1}+\left(\mathbf{S}^{-1}\right)^{\mathrm{T}} \mathbf{S}^{-1}\left(\lambda^{\max } \mathbf{I}+\mathbf{E}_{j}\right)=\mathbf{Q}
\end{aligned}
$$


The matrices $\mathbf{E}_{j}$ are continuous functions of $\boldsymbol{\delta}_{j}$, and their norms tend to zero uniformly on $j$ as $\delta_{0} \rightarrow 0$. This means that for a small enough value of $\delta_{0}$, matrix $\mathbf{Q}(3.6)$ is negative, and stability conditions (2.11) take place, which proves the first part of the theorem statement. Now let $\lambda_{P}^{\min }, \lambda_{P}^{\max }$ be the minimum and maximum eigenvalues of the matrix $\mathbf{P}$. It follows that inequality

$$
\lambda_{P}^{\min }\|\mathbf{x}\|^{2} \leqslant V(\mathbf{x}) \leqslant \lambda_{P}^{\max }\|\mathbf{x}\|^{2}
$$

is valid. Moreover, one can see that

$$
\mathbf{Q} \leqslant\left(\lambda^{\max }+\frac{1}{2} \widetilde{\lambda}\right)\left(\mathbf{S}^{-1}\right)^{\mathrm{T}} \mathbf{S}^{-1}+\left(\mathbf{S}^{-1}\right)^{\mathrm{T}} \mathbf{S}^{-1}\left(\lambda^{\max }+\frac{1}{2} \widetilde{\lambda}\right)=\left(2 \lambda^{\max }+\widetilde{\lambda}\right) \mathbf{P}
$$

Thus, $d V / d t \leqslant\left(2 \lambda^{\max }+\widetilde{\lambda}\right) V(t)$, and

$$
V(t) \leqslant V_{0} \exp \left[\left(2 \lambda^{\max }+\widetilde{\lambda}\right) t\right]
$$

Taking into account inequality (3.7), we conclude that (3.5) is fulfilled. This completes the proof of the theorem.

Corollary 3.1. It should be taken into account that in the second part of the theorem, the value $\lambda^{\text {max }}-\tilde{\lambda} / 2$ does not have to be negative. As it follows from (3.8), estimation (3.7) remains valid even if $\left\|\boldsymbol{\delta}_{j}\right\|$ is big enough, and the system becomes unstable.

Corollary 3.2. If for some period of time $[\widetilde{t}, \widetilde{t}+\widetilde{T}]\left(\widetilde{t} \geqslant t_{1}, \widetilde{T} \leqslant T\right)$ the maximum CLE of the output state vector is $\lambda_{\star}$, then for any small $\varepsilon>0$ there exist such switches that $\lambda_{j} \geqslant 2\left(\lambda_{\star}-\lambda^{\max }-\varepsilon\right)$.

The last acknowledgement allows one to construct a scheme for estimation the domain of values $\boldsymbol{\delta}_{j}$ for a given period of time, based on knowledge of the maximum CLE which is calculated from the information about the output signal.

Let us also note the fact that for some reasons it may be better to perform the linear transformation $\mathbf{x}=\mathbf{S z}$ first. Then equations (3.3) transform to

$$
\dot{\mathbf{z}}=\left(\mathbf{J}+\mathbf{S}^{-1} \mathbf{E}_{j} \mathbf{S}\right) \mathbf{z}
$$

Generally, $\mathbf{z}$ is a complex vector, but the Lyapunov function $V=\mathbf{z}^{\star} \mathbf{z}$ and its derivative

$$
\frac{d V}{d t}=\mathbf{z}^{\star}\left(\mathbf{J}^{\star}+\mathbf{S}^{\star} \mathbf{E}_{j}^{\star}\left(\mathbf{S}^{-1}\right)^{\star}+\mathbf{J}+\mathbf{S}^{-1} \mathbf{E}_{j} \mathbf{S}\right) \mathbf{z}
$$

are real. The form of notation by equations (3.9) may be more preferable when the matrix $\mathbf{S}$ (which is found by programme) is bulky and $\mathbf{S}^{-1}$ is even worse, then dealing with the matrix $\mathbf{P}$ may generate essential computational errors. For sure, one may overcome such a problem, but this will require additional effort, for instance appropriate scaling for the eigenvectors of $\mathbf{A}$.

Remark 3.1. Equations (3.9) can be easily reduced to the real form if, instead of the pair complex conjugate eigenvectors, a pair with real and imaginary parts is taken. Then the vector $\mathbf{z}$ becomes real, and the block with pair conjugate eigenvalues in the Jordan normal form is replaced as follows

$$
\left[\begin{array}{cc}
e i g_{s} & 0 \\
0 & \overline{e i g}_{s}
\end{array}\right] \longrightarrow\left[\begin{array}{cc}
\text { Re eig } & \text { Imeigs } \\
-\operatorname{Im} e i g_{s} & \text { Re eig }
\end{array}\right]
$$




\section{2-Degree of freedom system: Scheme of the fault estimation}

Let the original system (without control) linearized in the neighborhood of an unstable equilibrium is associated with the matrices of second order $\mathbf{M}, \mathbf{G}_{0}, \mathbf{H}_{0}$. To stabilize it, we introduce the control $\mathbf{u}(t)=\left[u_{1}(t), 0\right]^{\mathrm{T}}, u_{1}(t)=k_{1} q_{1}+k_{2} q_{2}+k_{3} \dot{q}_{1}+k_{4} \dot{q}_{2}$ and choose the appropriate values for $k_{s}(s=\overline{1,4})$. Once we wish to stabilize the system, the desired choice gives rather a big value to $\left|\lambda^{\max }\right|$ with the aim to reduce the stabilization period of time. But for our purpose (fault estimation) the rather small value for $\left|\lambda^{\max }\right|$ is more suitable. This makes easier the estimation of the maximum LCE of the output solution. Now we have a nominal (asymptotically stable) system with matrices

$$
\mathbf{G}=\mathbf{G}_{0}-\left[\begin{array}{cc}
k_{3} & k_{4} \\
0 & 0
\end{array}\right] \quad \mathbf{H}=\mathbf{H}_{0}-\left[\begin{array}{cc}
k_{1} & k_{2} \\
0 & 0
\end{array}\right]
$$

According to the previous Section, we consider faults related to the control effort as

$$
\mathbf{f}(t) \approx k_{1} \delta_{1}(t) \widetilde{q}_{1}+k_{2} \delta_{2}(t) \widetilde{q}_{2}+k_{3} \delta_{1}(t) \dot{\widetilde{q}}_{1}+k_{4} \delta_{2}(t) \dot{\widetilde{q}}_{2}
$$

where $\delta_{1}(t), \delta_{2}(t)$ are unknown piecewise functions.

The first step is to find the transformation matrix $\mathbf{S}$ and bring the equations to form (3.3) or, most likely, (3.9). If the system order is not very high, the corresponding analytical (due to presence uncertain parameters $\delta_{s}$ ) calculations are rather simple.

The second step comes from the study of (3.10). It derives the negativeness of the matrix

$$
\mathbf{D}\left(\lambda, \delta_{1 j}, \delta_{2 j}\right)=\lambda \mathbf{I}_{4}+\frac{1}{2}\left(\mathbf{S}^{\star} \mathbf{E}_{j}^{\star}\left(\mathbf{S}^{-1}\right)^{\star}+\mathbf{S}^{-1} \mathbf{E}_{j} \mathbf{S}\right)
$$

where $\lambda$ is another parameter which represents some upper bound for $\lambda^{\text {max }}$. For a 2-DOF system, this condition determines two or three 3D-surfaces that have the second order on $\delta$ and up to the fourth order on $\lambda$. For different values of $\lambda$, they determine contour lines on $\delta$-plane.

The third step relies upon calculation of the maximum CLE value for a given time interval and is based on measurement of the output signal - the time series for components of the state vector. Then, depending of the values found, the estimation of the corresponding $\delta$-domain is given.

\subsection{Algorithm}

Step 1. Construct a stabilizable control $\mathbf{u}(t)$ for system (2.8).

Step 2. Construct the matrix $\mathbf{S}$ from the Jordan normal form $\mathbf{J}=\mathbf{S}^{-1} \mathbf{A S}$.

Step 3. Fix the allowed maximum fault.

Step 4. Study the negativeness of the matrix $\mathbf{D}$ (4.3) with regard to $\lambda$.

Step $4 \mathrm{a}$. Find the time interval where the bound is exceeded.

\subsection{Illustrative example}

Below, we illustrate this procedure on the following example. Consider system (2.8) with

$$
\mathbf{M}=\left[\begin{array}{ll}
2 & 1 \\
1 & 1
\end{array}\right] \quad \mathbf{G}_{0}=\mathbf{0}, \quad \mathbf{H}_{0}=\mathbf{H}_{\Pi}+\mathbf{H}_{C}=\left[\begin{array}{ll}
8 & 2 \\
2 & 1
\end{array}\right]+\left[\begin{array}{cc}
0 & 9 / 5 \\
-9 / 5 & 0
\end{array}\right]
$$

The matrix of potential forces $\mathbf{H}_{\Pi}$ is positive definite, but the circulatory force makes the system unstable - eigenvalues are complex and two of them belong to the right half plane.

To stabilize the equilibrium, we introduce the control $u_{1}(t)$ and choose the appropriate values for $k_{s}:\left(-\frac{1199}{100},-\frac{23}{4},-\frac{681}{20},-\frac{613}{20}\right)$. 
Thus we have (4.1)

$$
\mathbf{G}=\left[\begin{array}{cc}
\frac{681}{20} & \frac{613}{20} \\
0 & 0
\end{array}\right] \quad \mathbf{H}=\left[\begin{array}{cc}
\frac{1999}{100} & \frac{191}{20} \\
\frac{1}{5} & 1
\end{array}\right] \quad \mathbf{A}_{0}=\left[\begin{array}{cccc}
0 & 0 & 1 & 0 \\
0 & 0 & 0 & 1 \\
-\frac{1979}{100} & -\frac{171}{20} & -\frac{681}{20} & -\frac{613}{20} \\
\frac{1959}{100} & \frac{151}{20} & \frac{681}{20} & \frac{613}{20}
\end{array}\right]
$$

The eigenvalues of the stabilized system are: $\mu_{1,2}=-\frac{1}{5} \pm 3 i, \mu_{3}=-1, \mu_{4}=-2$. Then the transformation matrix is as follows

$$
\mathbf{S}=\left[\begin{array}{cccc}
\frac{25}{21} & \frac{5}{3} & 1 & 0 \\
-1 & -1 & \frac{44481}{40501} & \frac{600}{40501} \\
-\frac{50}{21} & -\frac{5}{3} & -\frac{1}{5} & 3 \\
2 & 1 & \frac{35481}{202505} & \frac{133563}{40501}
\end{array}\right]
$$

and the expressions for blocks of the matrix $\mathbf{E}$ comes from (4.2)

$$
\mathbf{E}_{11}=\mathbf{E}_{12}=\mathbf{0} \quad \mathbf{E}_{21}=\left[\begin{array}{cc}
-\frac{1199}{100} \delta_{1} & -\frac{23}{4} \delta_{2} \\
\frac{1199}{100} \delta_{1} & \frac{23}{4} \delta_{2}
\end{array}\right] \quad \mathbf{E}_{22}=\left[\begin{array}{cc}
-\frac{681}{20} \delta_{1} & -\frac{613}{20} \delta_{2} \\
\frac{681}{20} \delta_{1} & \frac{613}{20} \delta_{2}
\end{array}\right]
$$

(a)

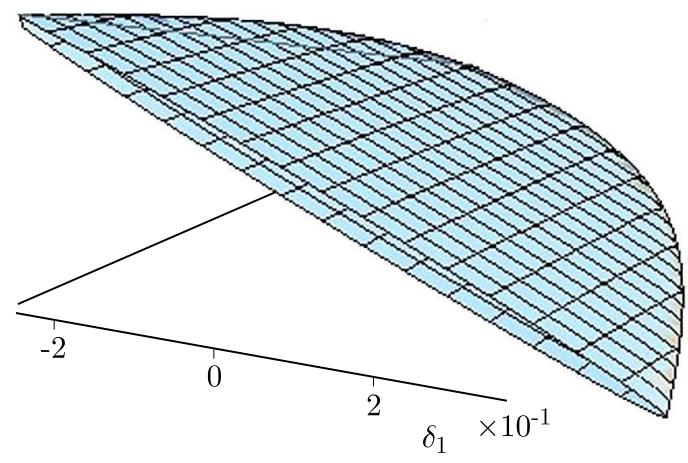

(b)

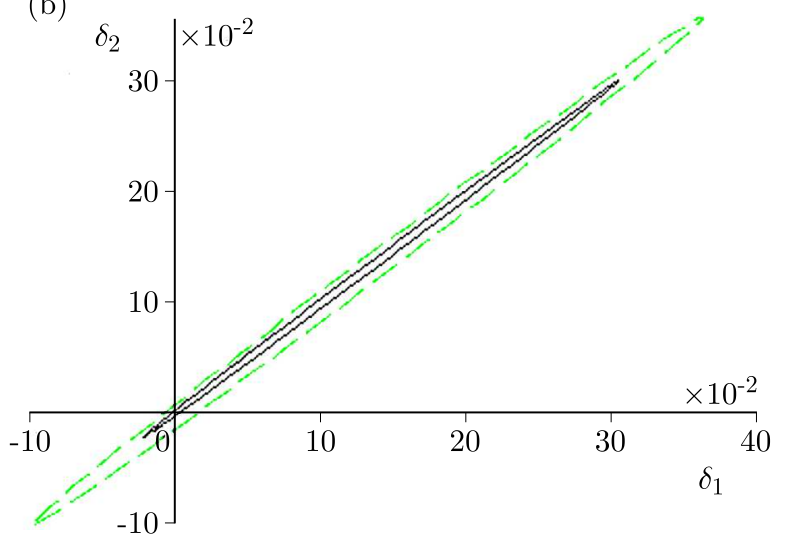

Fig. 2. Surface $\Delta_{4}=0$ and its cross-sections

Further we need to analyse the conditions of negativeness of the matrix D. For this purpose, since the matrix is of the fourth order, there is no real need to use the Schur complement. So, we use Sylvester's criterion which gives $\Delta_{2}>0, \Delta_{3}<0, \Delta_{4}=\operatorname{det} \mathbf{Q}>0$ (expressions for them are given in Appendix). The natural look of $\Delta_{4}=0$ is presented in Fig. 2a, and the contour lines with $\lambda=-0.1, \lambda=0$ in Fig. 2b. The shape of the surface is strongly flattened, and for better performance we shall give the scene the transformation

$$
\delta_{1}=\frac{1}{\sqrt{2}}\left(\widetilde{\delta}_{1}+0.02 \widetilde{\delta}_{2}\right) \quad \delta_{2}=\frac{1}{\sqrt{2}}\left(\widetilde{\delta}_{1}-0.02 \widetilde{\delta}_{2}\right)
$$

The resulting surface is presented in Fig. 3a. As one can see in Fig. 3b, the condition $\operatorname{det} \mathbf{D} \geqslant 0$ implies the fulfilment of $\Delta_{3} \leqslant 0$ - another surface "envelops" the first one. The condition $\Delta_{2} \geqslant 0$ is also satisfied, and the corresponding surface is much larger than the presented ones. Cross-sections of all three surfaces with the plane $\lambda=-0.1$ are shown in Fig. 4a. So, $\Delta_{4}>0$ is necessary and the sufficient condition for negativeness of $\mathbf{D}$. The contour lines (ellipses) for different values of $\lambda \geqslant-0.2$ are drawn in Fig. $4 \mathrm{~b}(\lambda=-0.15$ - dash line; $\lambda=-0.10$ - solid line; 


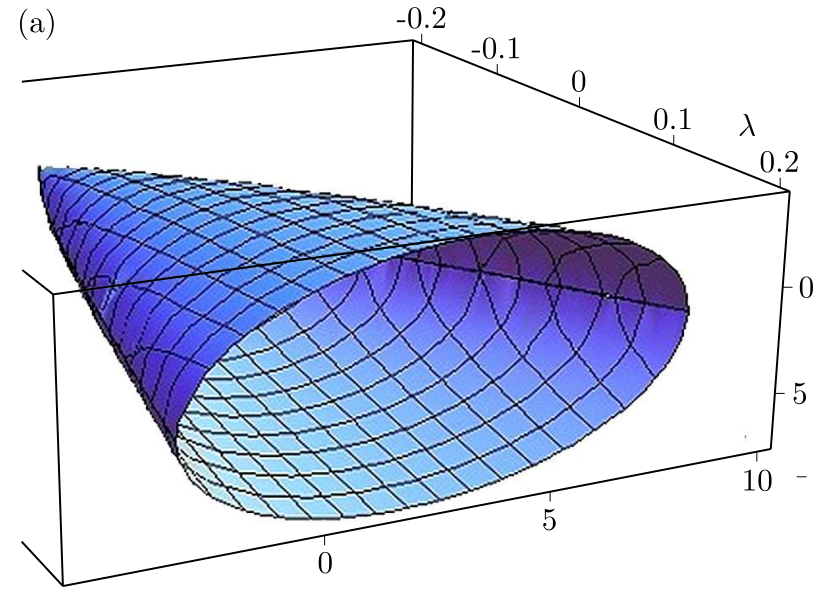

(b)

Fig. 3. Domain where the matrix $D$ is positive definite
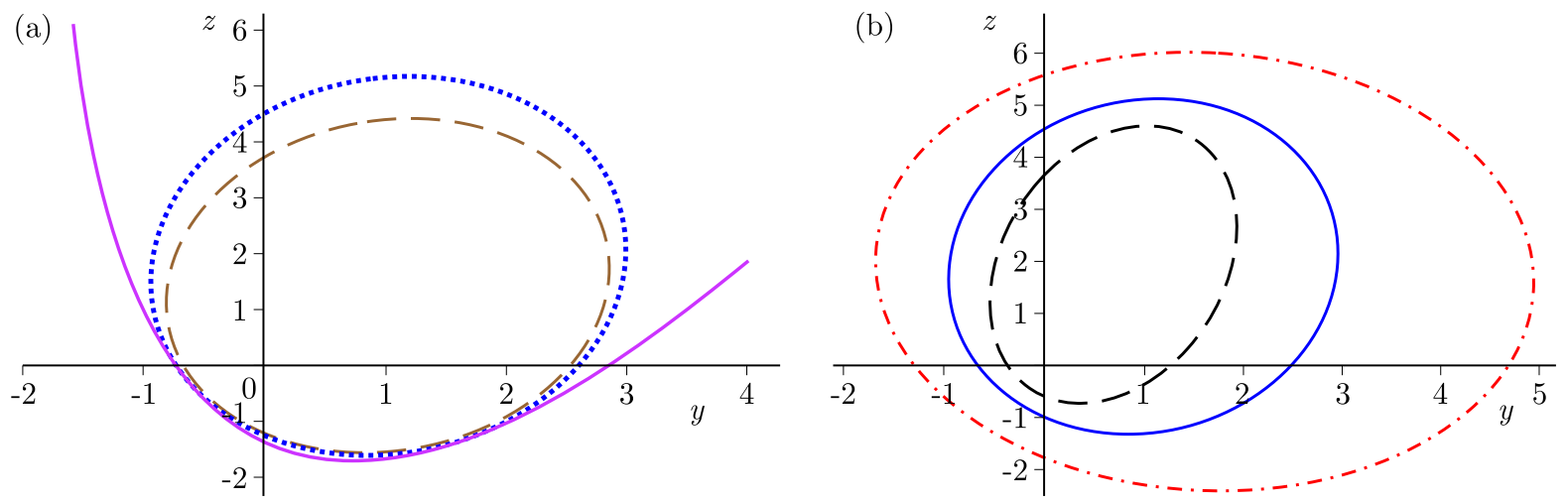

Fig. 4. Contour lines for different values of $\lambda$

(a)

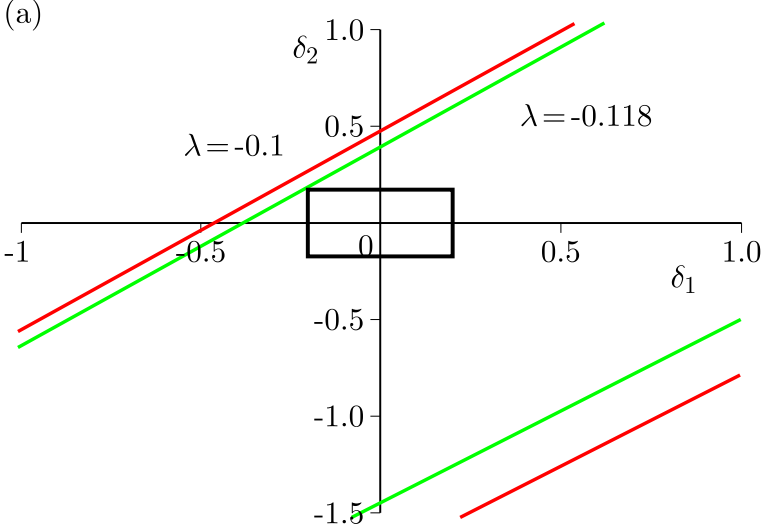

(b)

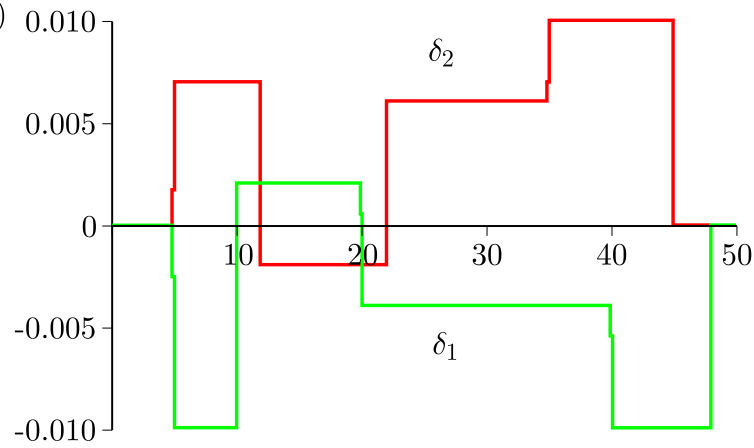

Fig. 5. (a) Maximum faults allowed; (b) functions $\delta_{s}$

$\lambda=0.05-$ dash dot line). Undoubtedly, all of them contain the origin. If $\lambda<-0.2$, there are hyperbolas, and the origin $\delta_{1}=0, \delta_{2}=0$ is situated between the branches.

There are several prerequisites for testing. Now suppose that the range of acceptable faults is given as $\left|\delta_{s}\right| \leqslant 0.002, s=1,2$; and the task is to estimate the time interval where this threshold value is exceeded. To find the corresponding value of $\lambda$, we can use the contour lines for $\Delta_{4}=0$ (Fig. 5a). The curve that has a common point with the black rectangle (and does not intersect it) is the one we are looking for, and $\lambda \approx-0.118$. After this, we will conduct a series of tests and gather the output of the state vector. The simulating results with piecewise functions $\delta_{s}$, 
$s=1,2$ (Fig. 5b) are presented in Fig. 6: the sample curve to the left and the output of the state coordinate to the right. Recall that for calculation of $\lambda^{\max }$, at least four tests with linearly independent initial conditions are necessary. So, Fig. 6 presents the clipped results. Obviously, the first time interval till $t \approx 10$ is not satisfactory - the decreasing of the amplitude is almost absent. The next interval (marked by snowflakes) $t \in[12,22]$ is more acceptable, and calculation of $\lambda^{\max }$ gives the value $\approx-0.115 \pm \varepsilon$. As a result, we can conclude that in this interval faults are small enough and satisfy the given limitations. In the next interval $[22,35]$, damping is also present, but the rate is insufficient $\lambda^{\max }>-0.1$. According to Section 3, this means that the values of $\delta_{s}$ (at least one of them) exceed the threshold level. The last interval is even easier the amplitude of the oscillation starts to increase.

(a)

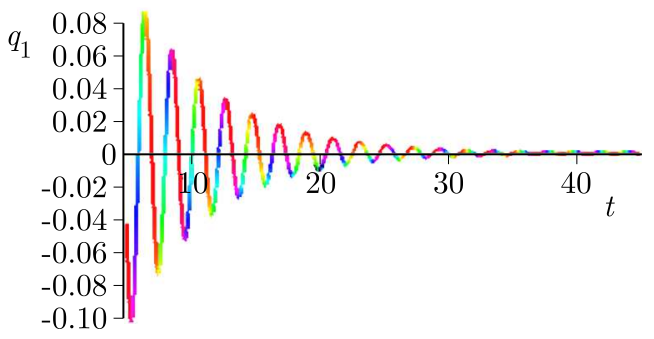

(b)

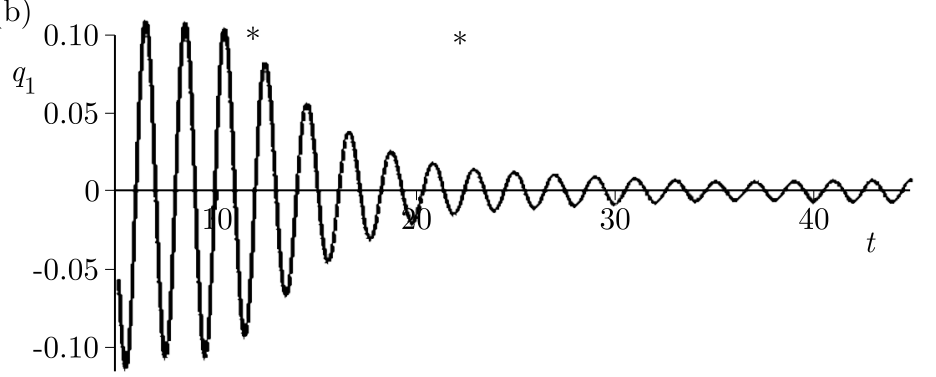

Fig. 6. State coordinate $q_{1}(t)$ sample and simulation curves

\section{Conclusion}

We have developed a scheme of fault detection for stabilizable underactuated dynamical systems. The approach is based on the estimation of the maximum Lyapunov exponent from the output of the state vector. We use the switching linear system model and the common quadratic Lyapunov function for the system under study. The theorem is given which establishes the link between the maximum CLE value of the perturbed solutions and the rate of the faults. One numerical example is given to illustrate the method.

The proposed approach seems to be a fairly simple and effective tool for identifying and estimating the errors that arise from inaccuracies in measuring the output signal and the effects of the actuator. When working with systems of second-order equations, it has certain advantages in constructing the Lyapunov function candidate (at least in comparison with the method of linear matrix inequalities), since it operates with matrices of lower orders. This allows one to reduce the time required for calculations and improve accuracy. As the subsequent objectives in the future study, the following targets can be noted:

- taking into account the influence of unknown disturbances ( $d$-terms in equations);

- increase the accuracy of the estimation due to additional analysis of the frequency component;

- weakening the restrictions on the nonlinear part of the equations of motion;

- testing the technique in laboratory experiments.

\section{Appendix}

Blocks of the matrix $\mathbf{Q}$

$$
\mathbf{Q}_{11}=\left[\begin{array}{cc}
-2-\frac{28055}{1224} \delta_{1}+\frac{7777}{408} \delta_{2} & -\frac{7721}{612} \delta_{1}+\frac{581}{68} \delta_{2} \\
\frac{28055}{3374} \delta_{1}+\frac{3333}{482} \delta_{2} & -1+\frac{1103}{241} \delta_{1}-\frac{747}{241} \delta_{2}
\end{array}\right]
$$




$$
\begin{aligned}
& \mathbf{Q}_{12}=\left[\begin{array}{cc}
\frac{1813}{1020} \delta_{1}-\frac{4464509}{13770340} \delta_{2} & \frac{4767}{136} \delta_{1}-\frac{190878611}{5508136} \delta_{2} \\
-\frac{777}{1205} \delta_{1}+\frac{5740083}{48803705} \delta_{2} & -\frac{6129}{482} \delta_{1}+\frac{245415357}{19521482} \delta_{2}
\end{array}\right] \\
& \mathbf{Q}_{21}=\left[\begin{array}{ll}
\frac{83183075}{6194664} \delta_{1}-\frac{3294115}{294984} \delta_{2} & \frac{32703955}{424476} \delta_{1}-\frac{246095}{49164} \delta_{2} \\
\frac{19801219}{2064888} \delta_{1}-\frac{3920719}{491640} \delta_{2} & \frac{3892487}{737460} \delta_{1}-\frac{292907}{81940} \delta_{2}
\end{array}\right] \\
& \mathbf{Q}_{22}=\left[\begin{array}{cc}
-\frac{1}{5}-\frac{153587}{14742} \delta_{1}+\frac{378207691}{1991191164} \delta_{2} & 3-\frac{673055}{32776} \delta_{1}+\frac{80850725945}{3982332328} \delta_{2} \\
-3-\frac{914011}{1229100} \delta_{1}+\frac{2250750323}{16593259700} \delta_{2} & -\frac{1}{5}-\frac{2403249}{163880} \delta_{1}+\frac{96230088317}{6637303880} \delta_{2}
\end{array}\right]
\end{aligned}
$$

Main diagonal minors of the matrix $\mathbf{Q}$

$$
\begin{aligned}
\Delta_{2} & =-1.0167 p_{2}^{2}+\left(-1.4688 \lambda+1.9956 p_{1}-0.2938\right) p_{2}+\lambda^{2}+\lambda\left(1.5706 p_{1}+0.4\right) \\
& +0.04-0.9792 p_{1}^{2}+0.3141 p_{1} \\
\Delta_{3} & =p_{2}^{2}(-1.7341 \lambda-1.1602)+p_{2}\left[\lambda\left(-1.6386+3.6541 p_{1}\right)-1.1589 \lambda^{2}-0.2814+2.3273 p_{1}\right] \\
& +\lambda^{3}+\lambda^{2}\left(1.1129 p_{1}+1.4000\right)+0.0400+\lambda\left(0.4400+1.7017 p_{1}-1.9501 p_{1}^{2}\right) \\
& +0.2958 p_{1}-1.1734 p_{1}^{2} \\
\Delta_{4} & =p_{2}^{2}\left(-4.4049 \lambda^{2}-7.3554 \lambda-2.7589\right)+p_{2}\left[-3.0650 \lambda^{3}+\lambda^{2}\left(9.3002 p_{1}-6.6249\right)\right. \\
& \left.+\lambda\left(15.1165 p_{1}-4.3973\right)-0.6390+5.5250 p_{1}\right]+\lambda^{4}+\lambda^{3}\left(3.4050 p_{1}+3.40000\right) \\
& +\lambda^{2}\left(3.2400+7.1364 p_{1}-5.0055 p_{1}^{2}\right)+\lambda\left(-7.8640 p_{1}^{2}+4.7076 p_{1}+0.9200\right) \\
& -2.7827 p_{1}^{2}+0.0800+0.6833 p_{1}
\end{aligned}
$$

\section{Acknowledgment}

This work was partially supported by the Spanish Ministry of Economy, Industry and Competitiveness, under grants DPI2016-77407-P (AEI/FEDER, 230 UE) and DPI2015-64170-R (MINECO/FEDER).

\section{References}

1. Chen J., Patton R.J., 1999, Robust Model-Based Fault Diagnosis for Dynamic Systems, Boston, Kluwer

2. Ding Z.X., 2008, Model-Based Fault Diagnosis Techniques, Springer

3. Eckmann J.-P., Ruelle D., 1992, Fundamental limitations for estimating dimensions and Lyapunov exponents in dynamical systems, Physica D, 56, 185-201

4. Frank P.M., 1990, Fault diagnosis in dynamic systems using analytical and knowledge-based redundancy, Automatica, 26, 459-474

5. Geromel J.C., Colaneri P., 2006, Stabilization of continuous-time switched linear systems, SIAM Journal on Control and Optimization, 45, 5, 1915-1930

6. Gertler J., 1998, Fault-Detection and Diagnosis in Engineering Systems, New York, Marcel Dekker

7. Hassibi A., Boyd S., 1998, Quadratic stabilization and control of piecewise-linear systems, Proceedings of the American Control Conference, Philadelphia, 3659-3664

8. Isermann R., 1997, Supervision, fault-detection and fault-diagnosis methods. An introduction, Control Engineering Practice, 5, 639-652

9. IsERmann R., 2005, Model-based fault detection and diagnosis-status and applications, Annual Reviews in Control, 29 71-85

10. Isermann R., 2011, Fault-Diagnosis Applications, Springer

11. LiBerzon D., 2003, Switching in Systems and Control, Birkhauser, Boston 
12. Liberzon D., Morse A.S., 1999, Basic problems in stability and design of switched systems, IEEE Control Systems Magazine, 19, 5, 59-70

13. Meskin N., Khorasani K., 2011, Fault Detection and Isolation: Multi-Vehicle Unmanned Systems, Springer

14. Molchanov A.P., Pyatnitskit E.S., 1989, Criteria of asymptotic stability of differential and difference inclusions encountered in control theory, Systems and Control Letter, 13, 1, 59-64

15. Muller P.C., 1995, Calculation of Lyapunov exponents for dynamic systems with discontinuities, Chaos, Solitons Fractals, 5, 9, 1671-1681

16. Muller P.C., 2009, Stability theory, [In:] Control Systems, Robotics and Automation. System Analysis and Control: Classical Approaches, H. Unbehauen (Edit.), vol. 3

17. Saberi A., Stoorvogel A.A., Sannuti T., 2007, Filtering Theory With Applications to Fault Detection, Isolation, and Estimation, Birkhauser, Boston

18. Shimada I., Nagashima T., 1979, A numerical approach to ergodic problem of dissipative dynamical systems, Progress of Theoretical Physics, 61, 6, 1605-1616

19. Shorten R.N., NArendra K.S., 1998, On the stability and existence of common Lyapunov functions for stable linear switching systems, Decision and Control, 4, 3723-3724

20. Talebi H.A., Abdollahi F., Patel R.V., Khorasani K., 2010, Neural Network-Based State Estimation of Nonlinear Systems: Application to Fault Detection and Isolation, Springer

21. Wolf A., Swift J.B., Swinney H.L., Vastano J.A., 1985, Determining Lyapunov exponents from a time series, Physica D, 16, 285

22. Xie G., Wang L., 2003, Controllability and stabilizability of switched linear-systems, Systems and Control Letter, 48, 2, 135-155

23. Zhai G., Hu B., Yasuda K., Michel A.N., 2000, Piecewise Lyapunov functions for switched systems with average dwell time, Asian Journal of Control, 2, 3, 192-197

24. Zhang W., EDIT., 2010, Fault Detection, In-tech 